\title{
Patterns of Organics on Substrates with Metallic Surface States: Why?, So??*
}

\author{
Theodore L. Einstein ${ }^{\dagger}$ \\ Department of Physics \& CMTC, University of Maryland, College Park, MD 20742-4111, U.S.A. \\ Ludwig Bartels \\ Department of Chemistry, University of California-Riverside, Riverside, CA 92521, U.S.A. \\ Josue R. Morales-Cifuentes \\ Department of Physics 85 CMTC, University of Maryland, College Park, MD 20742-4111, U.S.A.
}

(Received 16 February 2018; Accepted 1 April 2018; Published 25 May 2018)

\begin{abstract}
This paper modestly expands an invited talk at ISSS-8 with the same title. After reviewing the relevant interactions between adsorbates on substrates with metallic surface states [especially $\mathrm{Cu}(111)$ ], it focuses on organic adsorbates. Of particular interest are those which form honeycomb lattices with pores of various sizes. The nature of the confined states derived from the surface-state electrons is discussed as their effect on admolecules inside the pores. [DOI: 10.1380/ejssnt.2018.201]
\end{abstract}

Keywords: Surface electronic phenomena (work function, surface potential, surface states, etc.); Superlattices; Nano-particles, quantum dots, and supra-molecules; Friedel oscillation; Single crystal surfaces

\section{INTRODUCTION}

Superlattice patterns of adsorbates on surfaces have long provided an intriguing and fruitful aspect of surface science. From a practical standpoint, adsorbates are separated from their lateral neighbors so can be more active chemically. Until relatively recently, the adsorbates were atoms or small molecules. From a theoretical perspective, these superlattices provide one of the few physical examples of two-dimensional (2D) lattice-gas systems. Especially in the 1980 's, considerable interest was devoted to their phase transitions. For continuous transitions, the systems can be sorted into a relatively small number of universality classes, and the critical exponents are exactly simple rational fractions. Much attention was also given to unraveling the interaction energies between the adsorbates in lattice sites at various separations, which provide the physical underpinnings of these overlayers. Dipolar and elastic repulsions alone could not account for the diversity of patterns. Indirect interactions mediated by substrate conductions provide oscillatory (in sign) that can be anisotropic (when the electronic dispersion is) and are believed to play a central role in the formation of the superlattices.

More recently, patterns of organic molecules have generated considerable interest. The variety of structures is far greater for such adsorbates, and the periodicity is longer, often much longer, than for simple adatoms or admolecules. This large periodicity implies that the phase transitions will be first order, so that there are no critical properties to intrigue statistical mechanics. However, the large pores that form in many cases offer a host of fascinating and useful properties. Furthermore, the richness of the lateral interactions and geometries have long enticed engineer self-assembled patterns [1].

\footnotetext{
* This paper was presented at the 8th International Symposium on Surface Science, Tsukuba International Congress Center, Tsukuba, Japan, October 22-26, 2017.

† Corresponding author: einstein@umd.edu
}

\section{REVIEW OF INDIRECT INTERACTIONS}

The idea of an indirect interaction between adatoms is at least half a century old. Grimley [2] noted that pairs of atoms too far apart to overlap meaningfully could interact when adsorbed on a metal substrate and coupled to the occupied extended states of the metal. (See Ref. [3].) As their separation increases, the adsorbate wavefunctions will alternate between in-phase or out-of-phase coupling, corresponding to attractive or repulsive interactions, respectively. As the energy of the occupied substrate states increases, their wavelength will decrease. As the separation between the adatoms increases, a stationary-phase type argument shows that the behavior is dominated by the states at the Fermi level. In this asymptotic limit the interaction has the well-known form:

$$
E_{\text {pair }}(d) \propto d^{-n} \sin \left(2 q_{\mathrm{F}} d+2 \delta\right) \equiv d^{-n} \sin \left(4 d / \lambda_{\mathrm{F}}+2 \delta\right)
$$

which used to be called the Ruderman-Kittel-KosuyaYosida (RKKY) interaction [4-7]. (Magneticians now take RKKY to refer to all substrate-electron-mediated indirect interactions, not just the asymptotic limit in which the simple form is valid.) The phase factor $\delta$ is absent in the perturbative derivation. For many adsorption systems, the "black-scattering" (completely absorbing, no scattering to the bulk) limit is appropriate, with $\delta=\pi / 2$ $[8,9]$.

In the bulk it is also well known that $n=3$, but for mediation by the surface tails of bulk states, $n=5$, implying that the decay is so rapid that the magnitude is negligible by the separation that the asymptotic form is valid, confounding attempts to deduce lattice-gas interaction energies for Monte Carlo calculations to simulate experimental phase diagram. Also, the Fermi wavelength is very short.

When surface states mediate the interaction, $n=2$, and the asymptotic expression becomes physically significant. Most notably, for the (111) face of noble metals, there is a circular surface state at the center of the surface Brillouin zone, leading to circularly isotropic interactions with a Fermi wavelength of order $3 \mathrm{~nm}$. for $\mathrm{Cu}$ [10-12]. (These 
TABLE I. Shockley surface-state parameters for close-packed coinage-metal surfaces (and Si-Ag $\sqrt{3}$ ). The Shockley band is characterized by the effective electron mass $m_{\mathrm{eff}}$, the Fermi energy $E_{\mathrm{F}}$ measured relative to the bottom of the band $E_{0}$, and a corresponding in-surface Fermi wavevector $q_{\mathrm{F}}=\hbar^{-1} \sqrt{2 m_{\mathrm{eff}}\left(E_{\mathrm{F}}-E_{0}\right)}$ and half wavelength $\lambda_{\mathrm{F}} / 2=\pi / q_{\mathrm{F}}$. The Thomas-Fermi screening length, estimated from bulk parameters, $5.52 \mathrm{~nm}$ for $\mathrm{Cu}, 5.88 \mathrm{~nm}$ for $\mathrm{Ag}$ and $\mathrm{Au}$, and $\gg \lambda_{\mathrm{F}}$ for $\mathrm{Si}-\mathrm{Ag} \sqrt{3}$. For $\mathrm{S}, \mathrm{Cu}$, and $\mathrm{Co}$ on $\mathrm{Cu}$, the phase shift $\delta_{\mathrm{F}}$ is $\pm \pi / 2$ while for Co on $\mathrm{Ag}$ it is $\pi / 3$. Adapted from Refs. [9, 15], which give references for the entries.

\begin{tabular}{lcccc}
\hline \hline & $E_{\mathrm{F}}-E_{0}(\mathrm{eV})$ & $m_{\mathrm{eff}} / m_{\mathrm{e}}$ & $q_{\mathrm{F}}\left(\mathrm{nm}^{-1}\right)$ & 2.1 \\
$\mathrm{Cu}(111)-\mathrm{STM}$ & $0.38 / 0.39$ & 0.44 & 2.0 & 1.50 \\
$\mathrm{Cu}(111)-\mathrm{DFT}$ & 0.42 & 0.38 & $0.83 / 1.29$ & 1.55 \\
$\mathrm{Ag}(111)-\mathrm{STM}$ & $0.065 / 0.12$ & $0.40 / 0.53$ & & $3.79 / 244$ \\
$\mathrm{Ag}(111)-\mathrm{DFT}$ & 0.045 & & 1.73 & 1.82 \\
$\mathrm{Au}(111)-\mathrm{STM}$ & 0.41 & 0.28 & 0.10 & 3.1 \\
$\mathrm{Si}-\mathrm{Au} \sqrt{3}-\mathrm{STM}$ & 0.25 & 0.15 & & \\
\hline \hline
\end{tabular}

are, e.g., the source of Eigler's celebrated quantum corral STM pictures [13] and of oscillations near steps [14].) See Table I for relevant parameters.

Before moving on, it bears pointing out that in systems of current interest, the role of surface states becomes more subtle. For Dirac materials like graphene, the Fermi energy for undoped materials vanishes, which would lead to $\lambda_{\mathrm{F}} \rightarrow \infty$ behavior. Instead, $q_{\mathrm{F}}$ is replaced by an ultraviolet momentum cutoff for single-layer graphene $q_{\mathrm{c}}$ which is taken as the inverse lattice constant, so $4.07 \mathrm{~nm}^{-1}$ [16]. In another study, ordered arrangements of widely separated adatoms can be predicted [17]. If atoms couple to atop sites, then the exchange coupling between them decays like $d^{-3}$ [17-19] but with a different oscillatory factor and a prefactor that is $+1(-1)$ if the atoms are on the same (different) Bravais sublattices [10]. For undoped and doped graphene, a rich variety of analytic behaviors have been tabulated [20]. For bilayer graphene, which has quadratic rather than linear dispersion, behavior $d^{-2}$ is recovered [19, 21]. (For thicker graphene, there is a qualitative change in behavior [16].) On Weyl semimetals there are only Fermi arcs, disjointed 2D Fermi surfaces, implying no Friedel oscillations as in Eq. (1); however, if the same is thin, complementary Fermi arcs on the opposite side allow nested backscattering and strong Friedel oscillations [22]. Recent work [23, 24] on RKKY interactions between magnetic adatoms on Weyl semimetals find that there are four contributions from Heisenberg, Dzyaloshinsky-Moriya, spin-frustrated, and Ising couplings. A detailed discussion with implications for non-magnetic adatoms is beyond the scope of this proceedings paper.

\section{SIMPLE ORGANIC ADMOLECULES}

We use the case of benzene on $\mathrm{Cu}(111)$ as a prototypical example of an organic adsorbate system that does not differ greatly from adatomic adsorbates. This molecule has the 6 -fold point symmetry of the close-packed noble-metal top layer. Dougherty et al. [25] found two ordered hexagonal arrays, denoted C2 and C1 (see Fig. 1), with nearest neighbor spacings $0.69 \mathrm{~nm}$ and $1.024 \mathrm{~nm}$, corresponding to $8 / 3$ and 4 times the $\mathrm{Cu}(111)$ spacing $(0.255 \mathrm{~nm})$, re-

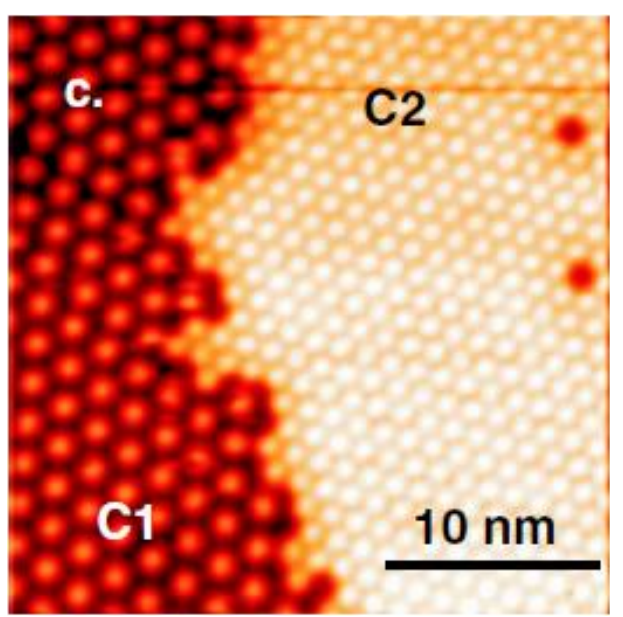

FIG. 1. Atomic-like ordered phases of benzene on $\mathrm{Cu}(111)$ : STM at 5 K. From Ref. [25].

spectively. The observation of clustering at subsaturation coverages suggests that these spacings correspond to attractions of the inter-benzene interaction. Indeed, the spacing $0.69 \mathrm{~nm}$ of the $\mathrm{C} 2$ phase corresponds to the minimum (at $32 \mathrm{meV}$ ) of the van der Waals interaction between a pair of planar benzene molecules with either an acene-like alignment or a mixed alignment. (For a phenyllike alignment, the minimum occurs at a large separation and is much less attractive.) The larger interadsorbate separation $(1.02 \mathrm{~nm})$ of the $\mathrm{C} 1$ phase is well beyond the minimum, implying that the van der Waals interaction is much weaker and not responsible for this sparser phase. Instead, we can attribute it to an indirect interaction mediated by the well-known metallic (partially filled) surface state on $\mathrm{Cu}(111)$, which has a minimum (albeit rather broad) near $1.02 \mathrm{~nm}$.

\section{HONEYCOMB NETS}

In contrast to the previous scenario, more linear organic molecules weakly adsorbed on the substrate can form honeycomb overlayers, with the admolecules forming the sides of the honeycomb cells. Some examples on $\mathrm{Cu}(111)$ in- 

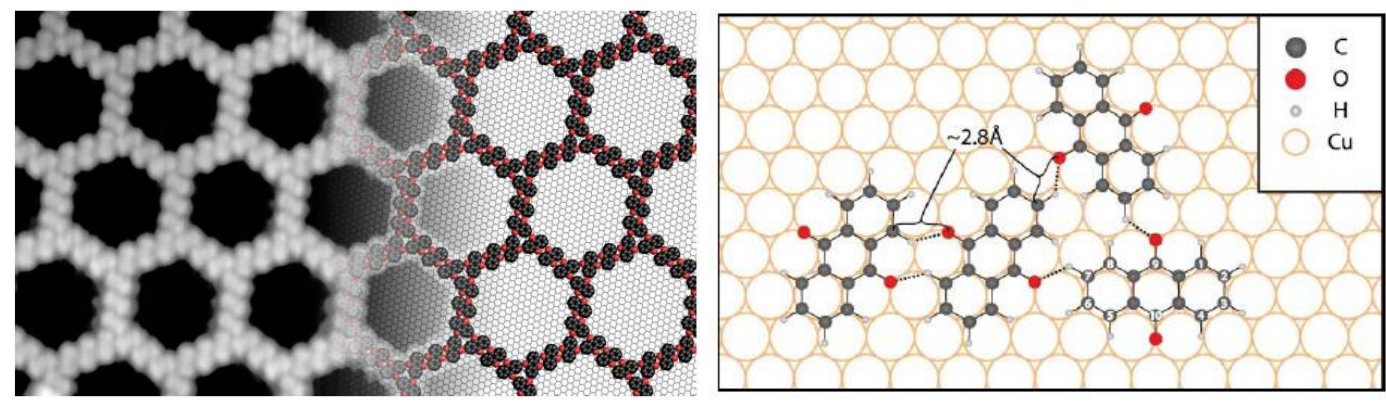

FIG. 2. Left panel (Left half) STM image of the honeycomb network of anthraquinone molecules on a Cu(111) surface. Bias, $1.3 \mathrm{~V}$; current, $73 \mathrm{pA}$; size, $26 \mathrm{~nm}$ by $15 \mathrm{~nm}$;.(Right half) a model of the $(\sqrt{304} \times \sqrt{304}) \mathrm{R} 23^{\circ}$ superstructure. Right panel: Model of the anthraquinone molecules forming a canted chain (left) and a vertex (right); $\mathrm{C}-\mathrm{H}-\mathrm{O}$ distances are indicated. From Ref. [31].

clude NC-Ph5-CN, 3deh-DPDI, and dehydro-DPDI [2629]; $\mathrm{Br}-\mathrm{DNT}$ on $\mathrm{Ag}(111)$ is another [30]. In all these cases, there is a single molecule on edge of the hexagonal pores. Arguably more intriguing is the case of anthraquinone (AQ) on $\mathrm{Cu}(111)$, for which there are 3 admolecules on each side [31, 32]. (See Fig. 2.) The high regularity of the overlayer was rather surprising given Villain's insight [33] for honeycomb arrays of domain boundaries for physisorbed systems. He noted that moving the vertices did not change the total length of the domain walls. Since this total length and the conserved number of vertices dominate the total wall energy, one would expect great irregularity in the honeycomb structures to enhance entropy. Their absence for $\mathrm{AQ} / \mathrm{Cu}(111)$ suggests that some characteristic energy stabilizes the regular pattern.

The first hypothesis was that some surface-statemediated interaction favors the size of the regular honeycomb (i.e., the separation between opposite edges of the honeycombs. The large size (186 exposed Cu's in each pore) of the system challenged Monte Carlo simulations of the ordered phase in terms of lattice-gas interactions. Also, the pores are chiral and have only 3 -fold symmetry. Two types of approximations were used by different groups. In one orientational dependence of the $\mathrm{AQ}-\mathrm{AQ}$ interaction in terms of the relative orientation of the two AQ's was neglected [34]. In the other, a large fraction of the lattice sites was discarded [35]. Thus, the agreement with experiment, while welcome, was hardly compelling.

A greater problem was that subsequent experiments showed that pentaquinone (PQ) forms the same ordered structure (albeit less robustly) [36]. See Fig. 3. Since the PQ molecules are larger than the AQ's, the distance across the honeycomb cell is larger for PQ admolecules. This renders suspect any explanation in terms of indirect interactions (based on the same $\mathrm{Cu}$ surface state). Hence, we proposed an alternative explanation: the structure was stabilized by electrons confined within the honeycomb cell [36]. We made the simplifying assumption that the barriers between cells were infinitely high, so that intercell coupling could be ignored in this zeroth approximation. Then we could invoke Fock's results [37] from 90 years (!) ago for electronic states confined in a $2 \mathrm{D}$ circular or square well. To determine the optimal configuration of a pore, we found the energies of the orbitals in the well as a function of well shape (how many AQ spacings per side, whether the hexagon is regular or otherwise, etc.) and populated these orbitals with the electrons that would have been in
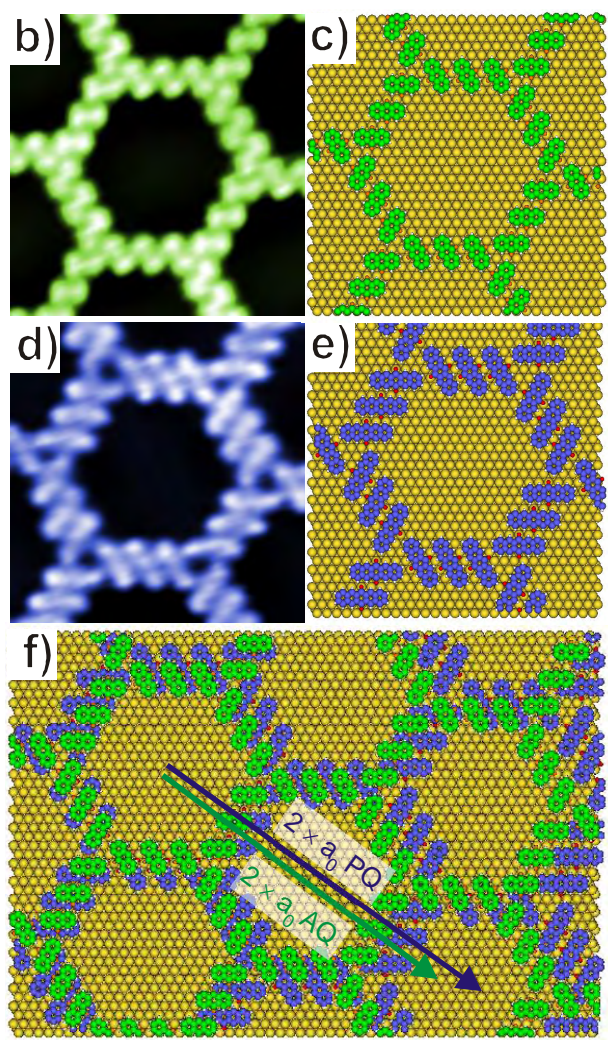

FIG. 3. STM image of parts of regular AQ network. (b, c) Single pore of AQ (image parameters: $7.4 \mathrm{~nm} \times 7.4 \mathrm{~nm}$; bias $2.4 \mathrm{~V}$; current $0.08 \mathrm{nA}$ ) andcorresponding model. (d, e) PQ pores (image parameters: $19 \mathrm{~nm} \times 9 \mathrm{~nm}$; bias $3.8 \mathrm{~V}$; current $0.01 \mathrm{nA}$ ) and corresponding model. (f) Superposition of PQ and AQ networks. The arrows show that the periodicities of the $\mathrm{AQ}$ and $\mathrm{PQ}$ networks differ substantially but their pore sizes are the same. From Ref. [36].

the surface state. For a simple circularly isotropic surface state with quadratic dispersion, this number (per [pore] area) is

$$
m_{\mathrm{eff}}\left(E_{\mathrm{F}}-E_{0}\right) / \pi \hbar^{2} .
$$

In using this expression, it is crucial that difference of the Fermi energy $E_{\mathrm{F}}$ and the minimum of the quadratic dispersion $E_{0}$ be adjusted to take into account charge transfer between the substrate and the AQ, which leads to an 
electric field $E$ normal to the substrate the magnitude of which should increase roughly linearly with coverage. (In some cases, such charge transfer is reframed as creating dipoles that lead to repulsions between admolecules [38]. This effect did not play any evident role in our study.)

Using a clever scheme for slab calculations, sketched in the appendix, Berland et al. [39] showed that such an $E$ shifts the Fermi energy relative to the band minimum by an energy $0.0146(7)[e \mathrm{~nm}] E$ for $\mathrm{Cu}(111)$. The shifts at higher coverages $(1 / 2-1 \mathrm{ML})$ predicted theoretically with this formula lie on the same line as the shifts measured with angular-resolved photoemission (ARPES) at the ALS in Berkeley. (Note that the powerful KorringaKohn-Rostoker Green's function (KKR-GF) method developed by Stepanyuk and coworkers avoids the problem of mixing between surface states on opposite sides of slabs by being able to treat a semi-infinite substrate, including with an applied normal external field $[40,41]$. It has also been used extensively to study magnetic interactions on surfaces.)

Wyrick et al.'s tabulations [36] considered pores with 3-6 AQ's per side. In each case, the regular (equal-sided) configuration had the lowest energy and of the four groupings, the case of 3 AQ's was the lowest. Furthermore, the 3-AQ regular case had exactly the correct number of electrons in the pore (when $E$ was included) to just fill the two molecular orbitals. (Likewise, for PQ, there are again just the right number of surface-state electrons in the pore to fill the two molecular orbitals, once a suitable shift of $E_{0}$ is made.) This situation is reminiscent of Kouwenhoven et al.'s [42] study of 2D quantum dots. That paper presented a periodic table of $2 \mathrm{D}$ artificial atoms. The "element" Oo (Oosterkampium, the "elements" being named after members of his group) corresponding to a 2D closedshell "noble gas" element with 2 electrons in the lowest circular level and 4 in the second level. (For the first two orbitals, Fock [37] showed that the shape difference from a square does not alter matters.) While for the dots one needs to consider Coulomb repulsions $e^{2} / C$, but with the metal surface the capacitance $C$ is essentially infinite and so insignificant for splitting the energies of the confined-electron orbitals. Note the distinction from typical quantum wells, in which the confinement creates and stabilizes the trapped orbitals. In this case, one can view the closed-shell orbitals as stabilizing the configuration of the confining honeycomb walls.

The other noble (coinage) metals have the same metallic surface state on the close-packed surfaces but with different $m_{\text {eff }}$ and $\left(E_{\mathrm{F}}-E_{0}\right)$. Thus, if the preceding analysis is valid, it would be surprising to find a giant regular honeycomb array of $\mathrm{AQ}$ on $\mathrm{Ag}(111)$ or $\mathrm{Au}(111)$. Experiments carried out by DeLoach et al. [43] on $\mathrm{Au}(111)$ confirmed this belief. There is no trace of honeycomb cells, let alone a regular network. While pairs of AQ's nestle into each other at an angle, as on $\mathrm{Cu}(111)$, the AQ's at low coverage form small clusters of 3 pairs arranged in a pinwheel. Note that using Eq. (2) and including the ratio of the areas (so the squared lattice constants of $\mathrm{Au}$ and $\mathrm{Cu}$ ), we expect there are only $88.5 \%$ of any cell on $\mathrm{Au}$ compared to $\mathrm{Cu}$. (Note also that at higher coverage, the AQ's form large disordered structures with pores satisfying an unusual area distribution associated with multiplicative noise.)

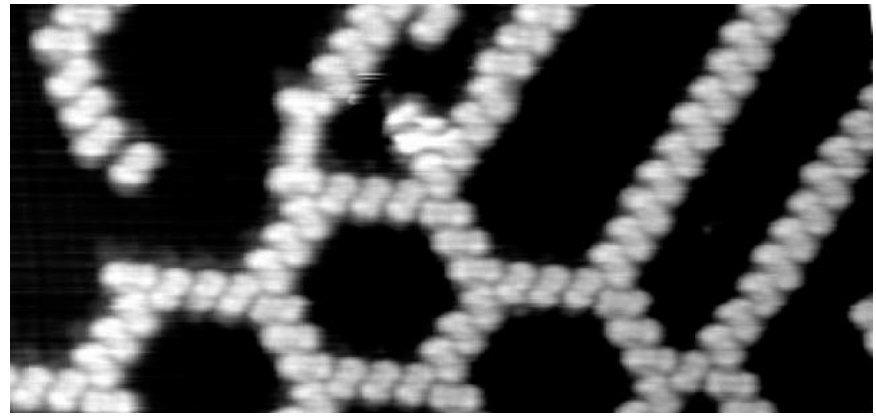

FIG. 4. Unpublished image of linear chains of $\mathrm{AQ}$ on $\mathrm{Cu}(111)$ at coverages somewhat below the threshold for the ordered honeycomb array. Annealed at room temperature after deposition (and prior to imaging at) liquid nitrogen temperature. See also Ref. [32] and supplementary Fig. S3 of Ref. [36].

\section{COMPARISON WITH OTHER HONEYCOMB STRUCTURES}

Lobo-Checo et al. [26] observed a large regular honeycomb of DPDI on $\mathrm{Cu}(111)$. With scanning tunneling spectroscopy (STS) they found a new peak $0.22 \mathrm{eV}$ below threshold characteristic of a confined dot state. Modest dispersion of this state was seen in ARPES experiments. For a similar system a pronounced confined state is seen at $0.73 \mathrm{NF}$ for single wall and double-wall honeycomb arrays (with modestly larger pores). Again, dispersion is observed, somewhat more for single-wall system. For AQ adsorbates, there is no evidence of dispersion in ARPES; this has been attributed to the great sensitivity of the giant honeycomb net to just the proper local coverage [36]. At low coverages, there are only strands of AQ's (see Fig. 4) and at slightly higher coverage than saturation (of the net), there are many irregular cells. A 20\% variation is enough to destroy the network. So only a small fraction of the overlayer has the characteristic honeycomb network. ${ }^{1}$

Another way to tune cell size is to use different lengths of, the same rod-like molecule. In a sense, this is similar

1 Linear (one-dimensional) arrays were seen earlier for pentacene on $\mathrm{Cu}(110)$ [44], but in that case the alignment was enhanced by the substrate anisotropy, with the admolecules parallel to each other (axis along [1ㅣㅣ]) rather than canted. More importantly, without the hydrogen bonds in pentaquinone, interactions between neighboring chains are insignificant, with chain formation attributed to lateral pressure to achieve densest packing. More germanely, $\mathrm{Co}$ on $\mathrm{Cu}(111)$ forms long chains; the separation between Co atoms is both the nearest-neighbor distance and the spacing of the first minimum in the calculated Co-Co lateral interaction. Calculation of the diffusion barriers indicate that the adatoms will preferentially attach to the ends rather than forming a compact cluster [45]. Thus, the observed self-assembled structure is not the equilibrium configuration. Indeed, it is quite a challenge to account for a chain structure with just pairwise interactions, while with trio (3-adatom nonpairwise) interactions [46] such chains can be readily explained. On Au(111) chains of TTF molecules follow the chevrons of the domain walls [47]. Last, we mention the formation of chains of Co or Fe atoms on $\operatorname{Ag}(111)$. Consistent with the long Fermi wavelength on $\operatorname{Ag}(111)$ (cf. Table I), the atoms are some 8 nearest-neighbor spacings apart, with the chain structure produced by biomolecular (methionine) nanograting [48]. 


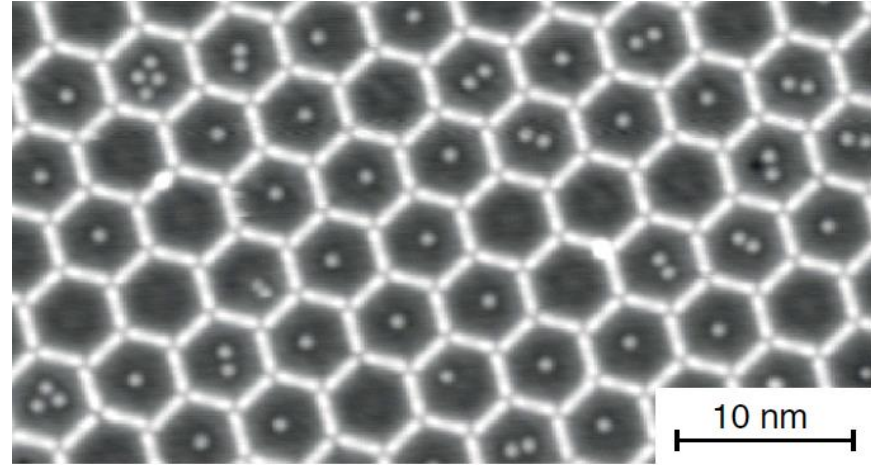

FIG. 5. Fe atoms in pores of NC-Ph5-CN on $\mathrm{Cu}(111)$ before annealing. From Ref. [29].

to substituting PQ for AQ. Brune and coworkers [29, 30] used different-length linear polyphenyl molecules, specifically chains of 3 and of 5 phenyl rings (coupled with $\mathrm{Cu}$ atoms from the substrate). They discuss the differences between the irregular honeycombs in the two cases in terms of a competition between admolecule-substrate and admolecule-admolecule interactions. They discuss the tilt of the central phenyl ring but mention nothing about states confined in the honeycomb pores.

\section{APPLICATIONS OF THE ARRAY OF PORES}

Having a network of identical pores offers the possibility of monitoring simultaneously the evolution of a vast number of equivalent systems. Arguably the nicest illustration of such behavior is the experiment by Pivetti et al. [29], see Fig. 5, in which Fe atoms such systems offer the experimental equivalent of doing massively parallel processing in Monte Carlo and similar computer simulations. Arguably the nicest illustration of such behavior is the experiment by Pivetti et al. [29], see Fig. 5, in which Fe atoms were placed in the honeycomb pores (containing 293 sites) of NC-Ph5-CN on $\mathrm{Cu}(111)$. They were able to monitor the distribution of the distances of the Fe atoms from the center of cells in which there were $1,2,3$, or many Fe atoms deposited at $12 \mathrm{~K}$. The distance distribution was narrower than that reported for adatom superlattices on homogeneous surface, consistent with an increase of surface-state mediated interactions due to confinement [49-51]. When the surface was annealed to $18 \mathrm{~K}$, they observed increases in these distances due to clustering of the atoms into rings.

For the minimum-size pores on honeycombs formed by DPDI, where there is a single-level quantum dot, there is no room for more than one adsorbate. In contrast, for the giant honeycomb network with AQ, there are 186 exposed $\mathrm{Cu}$ sites. Many experiments were performed with carbon monoxide additives [52-54]. In Fig. 6(a, b), we display the local density of state tables for the two filled orbitals in the honeycomb "dot", the ground state and the doubly degenerate second state, respectively [52]. Occupation data was then collected for $1-5 \mathrm{COs}$ in a pore. For two of these cases, Fig. 6(c, d) contains plots of the probability of $\mathrm{CO}$ occupation for each of the $186 \mathrm{Cu}$ substrate atoms exposed in the pore. While somewhat reminiscent of states in quantum corals, these plots differ qualitatively in their 3-fold rather than ring ("quantum onion") symmetry, as well as the much smaller area than inside a corral [13, 55, 56]. Calculations using KKR-GF methods [54-56] could impressively account for the standing wave patterns in corrals. The calculations also revealed enhanced interactions between adatoms in such confined geometries. It would be very interesting to what such calculations would show for $\mathrm{CO}$ in the pores of the $\mathrm{AQ}$ network on $\mathrm{Cu}(111)$.

Subsequently, movies were taken of 2-3 CO's, 2022 CO's, and vacancies in a $\sqrt{3}$ overlayer of CO's. The distribution of the last show that the structure has some chirality. The AQ overlayer has only 3 -fold rather than 6 -fold symmetry. In order to have 3 equivalent sides decorated in the same way by COs of a saturated $\sqrt{3}$ overlayer, there must be a dislocation line, which is evident crossing from edge to edge across the middle. Furthermore, at $24 \mathrm{~K}$ about $40 \%$ of the lines have kinks. The dislocations allow other adatoms greater access to the Cos as well as to the $\mathrm{Cu}$ substrate, so could enhance chemical reactivity. Such effects become more pronounced for smaller pores since there is a larger length-to-area ratio.

The observed dislocation lines (see Fig. 7) are in constant motion below $30 \mathrm{~K}$, a remarkable result since $\mathrm{CO}$ on $\mathrm{Cu}(111)$ does not begin to diffuse till $\sim 33 \mathrm{~K}$ [57]. Since $\mathrm{CO}$ adatoms are confined in pores, one can monitor the diffusion rate as a function of a fixed number density. The dotted line in Fig. 8 shows the diffusivity from a few molecules per pore up to $1 / 3 \mathrm{ML}$ (with $1 \mathrm{ML}$ being the saturated $(\sqrt{3} \times \sqrt{3})$ R30 $0^{\circ}$ adlayer $)$, when site blockage becomes important. For simplicity and lack of contradictory evidence, any coverage dependence of the prefactor is neglected. Closer examination of the STM images reveals that, in addition to the number of COs, their position in the pore plays a role, with COs around the perimeter moving faster than in the center, thwarting efforts to do a complete Arrhenius evaluation. The enhanced diffusion of confined CO (cf. Fig. 8) can be attributed to a reduction in the diffusion barrier with coverage, as discussed in Ref. [52]. Ref. [52] also shows that vacancies are most often found near pore edges, even though dislocation lines tend to cross the center; the distribution is rather featureless for 20-22 COs.

More generally, Polak and Rubinovich [58, 59] analyzed how confinement modifies the equilibrium constant of reactions, enhancing (suppressing) it for exothermic (endothermic) reactions (due primarily to the reduced numbers of mixed reactant-product microstates in the confined geometry). Confined cells can also stabilize reactive intermediates and labile products.

\section{SYMMETRY-BREAKING ON GRAPHENE}

A long-time goal has been to break the A-B sublattice symmetry of graphene. A decade ago a DFT calculation [60] showed that (single-layer) graphene on latticematched h-BN opened a gap of $53 \mathrm{meV}$ because of the different field on the A and B sublattices. This was subsequently engineered experimentally [61]. There were two experiments at University of Maryland to try to achieve 

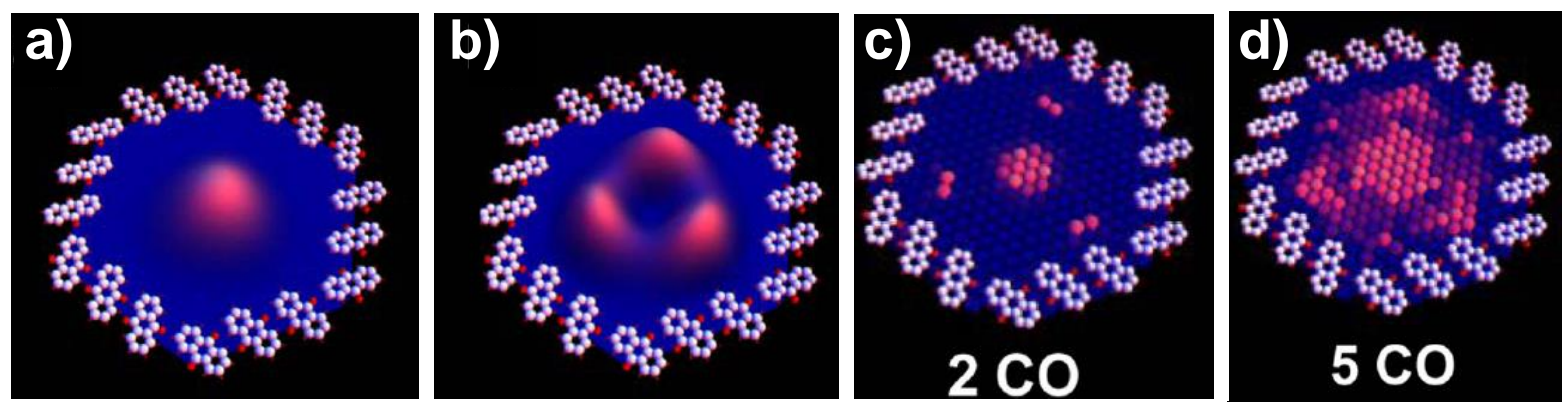

FIG. 6. (a, b) Plots of the calculated local DOS of (a) the lowest energy electronic state of the pore and (b) superposition of the two degenerate second electronic states of the pore. (c, d) Color-coded plots of the probability of CO molecule occupation for each of the $186 \mathrm{Cu}$ substrate atoms exposed within an AQ pore, for 2 and $5 \mathrm{COs}$, respectively. Each plot is based on over $500 \mathrm{CO}$ observed configurations, averaged over equivalent locations. Adapted from Ref. [52].

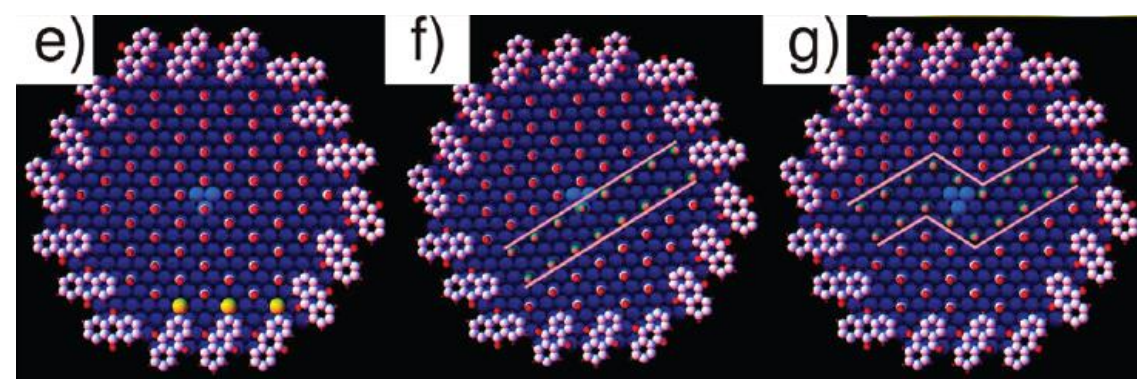

FIG. 7. Schematics of STM images at $24 \mathrm{~K}$ (panels b-d of Fig. 1 of Ref. [53]). (e, f) The $(\sqrt{3} \times \sqrt{3})$ R30 CO adlayer can be anchored at any one of the three atoms at the center of the exposed facet (light blue). In each case, one facet edge is decorated differently from the remaining two of the same kind [yellow in (e)]. (f) A dislocation line in the pore alleviates this difference without changing the number of molecules fitting inside the pore. $(\mathrm{g})$ Model of a kink in a dislocation line similar to the STM image of panel (d). Adapted from Ref. [53].

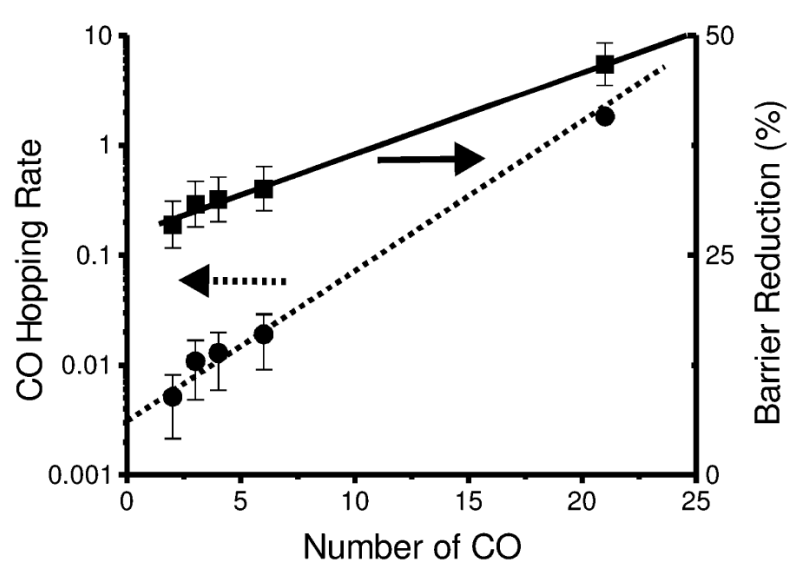

FIG. 8. Dotted line: diffusion rate per $\mathrm{CO}$ vs. number of molecules on an exposed facet. The last point was measured at $22 \mathrm{~K}$, the others at $27 \mathrm{~K}$. Solid line: reduction of the diffusion barrier that explains this acceleration, assuming a constant diffusion prefactor. All error bars are dominated by the $1 \mathrm{~K}$ uncertainty in measured temperatures; the statistical error is much smaller than the data markers [58].

similar effects with organic overlayers on graphene. The first were of a preliminary nature on graphite and used trimesic acid (TMA, or benzene-1,3,5-tricarboxylic acid) [62], which forms a rich variety of patterns [63]. The simplest honeycomb $\left(\mathrm{H}_{1}\right)$ has some two dozen exposed rings in a honeycomb pore. Depending on how the honeycomb is aligned relative to a graphene substrate, AB symmetry might or might not be broken. DFT calculations by Josue Morales-Cifuentes provided estimates of the splittings that might be expected [64].

The second set of experiments considered Freon-13 $\left(\mathrm{CClF}_{3}\right)$ adsorbates [65]. Wang et al. observed a dip in the conductivity at parameters corresponding to an ordered phase in the phase diagram. They accordingly speculated that the dip might be associated with the opening of a gap. The measurements spurred Morales-Cifuentes to carry out DFT calculations for four configurations of a $\mathrm{p}(2 \times 2)$ overlayer: with the $\mathrm{Cl}$ above a carbon atom or in the center of a honeycomb and with the $\mathrm{F}$ atoms pointing to the nearest carbon atoms or the middle of the bond between two of them. In some of these cases, gaps of several $\mathrm{meV}$ do open, when the triad of $\mathrm{F}$ atoms break sublattice symmetry, most noticeably when the $\mathrm{Cl}$ is above a graphene C atom. Details will be reported soon [64].

\section{SUMMARY}

With large organic molecules adsorbed lying down on metal substrates, it is now possible to form regular arrays of cells that contain confined electronic states. Into these pores one can adsorb smaller molecules and monitor configurations and reactions. By judicious choice of the molecules, one can in principle tailor the reactions to enhance. In some cases, termed here "self-sustained", 
the confined surface electronic states appear to actually stabilize the configuration: not only does the surface state get captured in the "corral", it drives the formation of the corral. We have discussed at length the giant honeycomb array of anthraquinone and pentaquinone on $\mathrm{Cu}(111)$, which appear to be prime examples of this behavior. More detailed calculations going beyond the simplifying assumptions of present work (e.g., no leakage of surface states between pores) will be needed to assess the validity of this picture (especially given the sensitivity of the stabilization) and to give a clearer since of which reactions will be especially enhanced. Likewise, further experiments could enrich the collection of systems with honeycomb arrays.

\section{APPENDIX: Calculation of surface states in slab systems [39]}

In some model calculations one can compute surface states using a semi-infinite solid. However, computing surface states in slab geometries, as is commonly done in DFT investigations, poses a special challenge because the states on opposite sides of the slab couple together to form bonding (antibonding) combinations with lower (higher) energies than the actual states. The resulting splittings become particularly vexing near the Fermi level, where it is most important to characterize the surface state accurately. In a $2 \times 2$ Hermitian matrix description, the change in the actual surface-state energy $\varepsilon(\boldsymbol{k})$ is due to a detuning $W(\boldsymbol{k})$ which shifts $\varepsilon$ up or down and a coupling $\Omega(\boldsymbol{k})$ between the states (in the off-diagonals). Then the energies of the antibonding (bonding) combinations of the coupled surface states are $\varepsilon \pm\left(W^{2}+\Omega^{2}\right)^{1 / 2}$, and the asso- ciated eigenfunctions can be found. Then, following the the MaxLoc criterion of Marzari and Vanderbilt [66], one determines $W$ and $\Omega$ to minimize the spread of the pair of eigenfunctions $\phi_{+}$and $\phi_{-}$:

$$
\Sigma_{i}\left|\left\langle\phi_{i}\left|z_{i}^{2}\right| \phi_{i}\right\rangle\right|-\left|\left\langle\phi_{i}\left|z_{i}\right| \phi_{i}\right\rangle\right|^{2}
$$

Since there is an inversion center, the Bloch functions are real, so only a rotation is needed.

Results for $W$ and $\Omega$ and $\varepsilon_{\mathrm{F}}$ are tabulated in Ref. [39]. The Fermi energy $\varepsilon_{\mathrm{F}}$ converges to the sub-meV level for a 15-layer slab with this decoupling method. For a six-layer slab, the surface-state energy differs from the converged value by $80 \mathrm{meV}$, about 20\%; Berland et al. concluded that 6 layers is the minimum slab thickness for an adequate approximate account of the surface state, which can be useful for studying surface-state shifts for adsorbatessystems requiring a large supercell in the in-plane direction.

\section{ACKNOWLEDGMENTS}

We acknowledge with gratitude the extensive collaboration with Kristian Berland, Per Hyldgaard; Daniel B. Dougherty, Andrew S. DeLoach, Brad R. Conrad; Zhihai Cheng, Jonathan Wyrick, Zhihai Cheng, Miaomiao Luo, and others in the Bartels's group. Our work was supported in part by NSF Grant No. CHE 13-05892 (TLE) and CHE 13-06969 (LB). Computer resources were provided by XSEDE/NSF (CHE 13-0106). We also thank the referee for calling our attention to several papers published in the preceding decade.
[1] T. Yokoyama, S. Yokoyama, T. Kamikado, Y. Okuno, and S. Mashiko, Nature 413, 619 (2001).

[2] T. B. Grimley, Proc. Phys. Soc. 90, 751 (1967).

[3] T. L. Einstein, in: Handbook of Surface Science, Vol. 1, edited by W. N. Unertl (Elsevier, Amsterdam, 1996).

[4] M. A. Ruderman and C. Kittel, Phys. Rev. 96, 99 (1954).

[5] T. Kasuya, Prog. Theor. Phys. 16, 45 (1956).

[6] K. Yosida, Phys. Rev. 106, 893 (1957).

[7] J. H. Van Vleck, Rev. Mod. Phys. 34, 681 (1962).

[8] P. Hyldgaard and M. Persson, J. Phys.: Cond. Matter 12, L13 (2000).

[9] P. Hyldgaard and T. L. Einstein, Europhys. Lett. 59, 265 (2002).

[10] S. D. Kevan, Phys. Rev. Lett. 50, 526 (1983).

[11] L. Petersen, P. Laitenberger, E. Lægsgaard, and F. Besenbacher, Phys. Rev. B 58, 7361 (1998).

[12] J. Repp, F. Moresco, G. Meyer, K.-H. Rieder, P. Hyldgaard, and M. Persson, Phys. Rev. Lett. 85, 2981 (2000).

[13] M. Crommie, C. Lutz, and D. Eigler, Nature 363, 524 (1993).

[14] M. M. Kamna, S. J. Stranick, and P. S. Weiss, Science 274, 118 (1996).

[15] P. Hyldgaard and T. L. Einstein, J. Cryst. Growth 275, e1637 (2005); P. Hyldgaard and T. L. Einstein, Surface Sci. 532-535, 600 (2003).

[16] H. Min, E. H. Hwang, and S. Das Sarma, Phys. Rev. B
95, 155414 (2017).

[17] V. V. Cheianov, V. I. Fal'ko, O. Syljuåsen, and B. L. Altshuler, Solid State Commun. 149, 1499 (2009).

[18] V. V. Cheianov and V. I. Fal'ko, Phys. Rev. Lett. 97, 226801 (2006).

[19] S. Das Sarma, S. Adam, E. H. Hwang, and E. Rossi, Rev. Mod. Phys. 83, 407 (2011); E. Kogan, Phys. Rev. B 84, 115119 (2011).

[20] M. Sherafati and S. Satpathy, Phys. Rev. B 84, 125416 (2011).

[21] E. H. Hwang and S. Das Sarma, Phys. Rev. Lett. 101, 156802 (2008).

[22] P. Hosur, Phys. Rev. B 86, 195102 (2012).

[23] M. V. Hosseini and M. Askari, Phys. Rev. B 92, 224435 (2015).

[24] H.-R. Chang, J. Zhou, S.-X. Wang, W.-Y. Shan, and D. Xiao, Phys. Rev. B 92, 241103(R) (2015).

[25] D. B. Dougherty, P. Maksymovych, J. Lee, and J. T. Yates, Jr., Phys. Rev. Lett. 97, 236806 (2006).

[26] J. Lobo-Checa, M. Matena, K. Müller, J. H. Dil, F. Meier, L. H. Gade, T. A. Jung, and M. Stöhr, Science 325, 300 (2009).

[27] N. Kepčija, T.-J. Huang, F. Klappenberger, and J. V. Barth, J. Chem. Phys. 142, 101931 (2015).

[28] I. Piquero-Zulaica, J. Lobo-Checa, Ali Sadeghi, Z. M. Abd El-Fattah, C. Mitsui, T. Okamoto, R. Pawlak, T. Meier, A. Arnau, J. E. Ortega, J. Takeya, S. Goedecker, 
E. Meyer, and S. Kawai, Nat. Commun. 8, 787 (2017).

[29] M. Pivetta, G. E. Pacchioni, U. Schlickum, J. V. Barth, and H. Brune, Phys. Rev. Lett. 110, 086102 (2013).

[30] G. E. Pacchioni, M. Pivetta, and H. Brune, J. Phys. Chem. C 119, 25442 (2015).

[31] G. Pawin, K. L. Wong, K. Y. Kwon, and L. Bartels, Science 313, 961 (2006).

[32] G. Pawin, Molecular Diffusion, Energetics, and Structures on Cu(111) As Studied by Scanning Tunneling Microscopy, Ph. D. thesis, University of California-Riverside, 2008. https://escholarship.org/uc/item/67n6928r

[33] J. Villain, in: Ordering in Strongly Fluctuating Condensed Matter Systems, edited by T. Riste (Plenum Press, New York, 1980) p. 221.

[34] K. Kim and T. L. Einstein, Phys. Rev. B 83, 245414 (2011).

[35] M. Šimėnas and E. E. Tornau, J. Chem. Phys. 139, 154711 (2013).

[36] J. Wyrick, D. H. Kim, D. Z. Sun, Z. H. Cheng, W. H. Lu, Y. M. Zhu, K. Berland, Y. S. Kim, E. Rotenberg, M. M. Luo, P. Hyldgaard, T. L. Einstein, and L. Bartels, Nano Lett. 11, 2944 (2011).

[37] V. Fock, Z. Phys. 47, 446 (1928).

[38] T. Yokoyama, T. Takahashi, K. Shinozaki, and M. Okamoto, Phys. Rev. Lett. 98, 206102 (2007).

[39] K. Berland, T. L. Einstein, and P. Hyldgaard, Phys. Rev. B 85, 035427 (2012).

[40] P. A. Ignatiev and V. S. Stepanyuk, Phys. Rev. B 84, 075421 (2011)

[41] P. A. Ignatiev, O. O. Brovko, and V. S. Stepanyuk, Phys. Rev. B 86, 045409 (2012).

[42] L. Kouwenhoven and C. Marcus, Phys. World 11, 35 (1998); L. P. Kouwenhoven, D. G. Austing, and S. Tarucha, Rep. Prog. Phys. 64, 701 (2001).

[43] A. S. DeLoach, B. R. Conrad, T. L. Einstein, and D. B. Dougherty, J. Chem. Phys. 147, 184701 (2017).

[44] S. Lukas, G. Witte, and Ch. Wöll, Phys. Rev. Lett. 88, 028301 (2001).

[45] V. S. Stepanyuk, A. N. Baranov, D. V. Tsivlin, W. Hergert, P. Bruno, N. Knorr, M. A. Schneider, and K. Kern, Phys. Rev. B 68, 205410 (2003).

[46] T. L. Einstein, Langmuir 7, 2520 (1991).

[47] I. Fernandez-Torrente, S. Monturet, K. J. Franke, J. Fraxedas, N. Lorente, and J. I. Pascual, Phys. Rev. Lett. 99, 176103 (2007).

[48] A. Schiffrin, J. Reichert, W. Auwärter, G. Jahnz, Y. Pennec, A. Weber-Bargioni, V. S. Stepanyuk, L. Niebergall, P. Bruno, and J. V. Barth, Phys. Rev. B 78, 035424
(2008).

[49] V. S. Stepanyuk, N. N. Negulyaev, L. Niebergall, R. C. Longo, and P. Bruno, Phys. Rev. Lett. 94, 187201 (2005).

[50] V. S. Stepanyuk, N. N. Negulyaev, L. Niebergall, R. C. Longo, and P. Bruno, Phys. Rev. Lett. 97, 186403 (2006).

[51] V. S. Stepanyuk, N. N. Negulyaev, L. Niebergall, and P. Bruno, New J. Phys. 9, 388 (2007).

[52] Z. Cheng, J. Wyrick, M. Luo, D. Sun, D. Kim, Y. Zhu, W. Lu, K. Kim, T. L. Einstein, and L. Bartels, Phys. Rev. Lett. 105, 066104 (2010).

[53] Z. Cheng, M. Luo, J. Wyrick, D. Sun, D. Kim, Y. Zhu, W. Lu, K. Kim, T. L. Einstein, and L. Bartels, Nano Lett. 10, $3700(2010)$.

[54] M. Luo, An STM Study of Molecular Self-Assemblies on Cu(111): Structure, Interaction, and Effects of Confinement, Ph. D. thesis, University of California-Riverside, 2012. https://escholarship.org/uc/item/67n6928r

[55] H. C. Manoharan, C. P. Lutz, and D. M. Eigler, Nature 403, 512 (2000).

[56] F. Silly, M. Pivetta, M. Ternes, F. Patthey, J. P. Pelz, and W.-D. Schneider, Phys. Rev. Lett. 92, 016101 (2004); F. Silly, M. Pivetta, M. Ternes, F. Patthey, J. P. Pelz, and W.-D. Schneider, New J. Phys. 6, 16 (2004).

[57] K. L. Wong, B. V. Rao, G. Pawin, E. Ulin-Avila, and L. Bartels, J. Chem. Phys. 123, 201102 (2005).

[58] M. Polak and L. Rubinovich, Nano Lett. 8, 3543 (2008).

[59] M. Polak and L. Rubinovich, Phys. Chem. Chem. Phys. 13, 16728 (2011).

[60] G. Giovannetti, P. A. Khomyakov, G. Brocks, P. J. Kelly, and J. van den Brink, Phys. Rev. B 76, 073103 (2007).

[61] Z. Liu, L. Ma, G. Shi, W. Zhou, Y. Gong, S. Lei, X. Yang, J. Zhang, J. Yu, K. P. Hackenberg, A. Babakhani, J.-C. Idrobo, R. Vajtai, J. Lou, and P. M. Ajayan, Nat. Nanotechnol. 8, 119 (2013).

[62] M. A. Groce, Organic Molecular Thin Films on DeviceRelevant Substrates, Ph. D. thesis, University of Maryland, 2013. https://drum.lib.umd.edu/handle/1903/ 14581

[63] Y. Ye, W. Sun, Y. Wang, X. Shao, X. Xu, F. Cheng, J. Li, and K. Wu, J. Phys. Chem. C 111, 10138 (2007).

[64] J. R. Morales-Cifuentes and T. L. Einstein, APS March Meeting (Baltimore, 2013) M6.00007; J. R. MoralesCifuentes, Y. Wang, J. Reutt-Robey, and T. L. Einstein, APS March Meeting (Denver, 2014) Q53.00008; and in preparation.

[65] Y. Wang, W. Bao, S. Xiao, M. S. Fuhrer, and J. ReuttRobey, Appl. Phys. Lett. 103, 201606 (2013).

[66] N. Marzari and D. Vanderbilt, Phys. Rev. B 56, 12847 (1997). 